\title{
TIME PERIODIC SOLUTIONS OF THE NAVIER-STOKES EQUATIONS WITH THE TIME PERIODIC POISEUILLE FLOW IN TWO AND THREE DIMENSIONAL PERTURBED CHANNELS
}

\author{
TEPPEI KoBAYASHI
}

(Received September 25, 2012, revised April 8, 2013)

\begin{abstract}
H. Beirão da Veiga proved that, for a straight channel in $\boldsymbol{R}^{n}$ ( $n$ arbitarily large) and for a given flux with the time periodicity, there exists a unique time periodic Poiseuille flow in a straight channel in $\boldsymbol{R}^{n}$. Furthermore, the existence of a time periodic solution in a perturbed channel (Leray's problem) is shown for the Stokes problem (arbitary dimension) and for the Navier-Stokes problem $(n \leq 4)$. Concerning the Navier-Stokes case, a quatitative condition requaired to show the existence of a time periodic solution depends not just on the flux of the time periodic Poiseuille flow but also on the domain it self. In this paper, by applying the result of H. Beirão da Veiga and C. J. Amick, we succeed in proving the independence of such a condition on the particular domain.
\end{abstract}

1. The time periodic Poiseuille flow. In this section, for a straight channel in $\boldsymbol{R}^{n}(n=$ 2,3 ), which is parallel to the $x_{1}$-axis, let us consider a time periodic flow of an incompressible viscous fluid which is also parallel to the $x_{1}$-axis.

In the case $n=2$, for $a>0$ we suppose $\Sigma:=(-a, a)$. In the case $n=3$, we suppose that $\Sigma$ is a bounded smooth simply connected domain in $\boldsymbol{R}^{2}$. We write

$$
\omega=\boldsymbol{R} \times \Sigma .
$$

Then $\omega$ is a straight and infinite channel, which is parallel to the $x_{1}$-axis, and $\Sigma$ is a cross section of the channel $\omega$.

In the straight and infinite channel $\omega$, let us consider the nonstationary Navier-Stokes equations

$$
\begin{gathered}
\frac{\partial \boldsymbol{u}}{\partial t}-v \Delta \boldsymbol{u}+(\boldsymbol{u} \cdot \nabla) \boldsymbol{u}+\nabla p=\mathbf{0} \quad \text { in } \boldsymbol{R} \times \omega, \\
\operatorname{div} \boldsymbol{u}=0 \quad \text { in } \quad \boldsymbol{R} \times \omega, \\
\boldsymbol{u}=\mathbf{0} \quad \text { on } \quad \boldsymbol{R} \times \partial \omega
\end{gathered}
$$

with the time periodic condition

$$
\boldsymbol{u}(t)=\boldsymbol{u}(t+T) \quad \text { in } \quad \omega
$$

2000 Mathematics Subject Classification. Primary 35Q30; Secondary 14F40, 76D05.

Key words and phrases. Time periodic solutions of the Navier-Stokes equations, the Poiseuille flow, channels. 
and the flux condition

$$
\int_{\Sigma} \boldsymbol{u}(t) \cdot \boldsymbol{n} d S=\alpha(t) \quad(t \in \boldsymbol{R}),
$$

where $\boldsymbol{u}=\boldsymbol{u}(t, x)$ and $p=p(t, x)$ are the unknown velocity and the unknown pressure of the fluid motion in $\omega$, respectively, $v$ is the given viscosity constant, $T(>0)$ is a given constant, $\boldsymbol{n}$ is the unit parallel vector to the $x_{1}$-axis and $\alpha(t)$ is a given $T$-periodic real function.

Since we look for a solution pallalel to the $x_{1}$-axis, we may assume that

$$
\begin{array}{ll}
\boldsymbol{u}(t, x)=(v(t, x), 0) & (n=2), \\
\boldsymbol{u}(t, x)=(v(t, x), 0,0) & (n=3) .
\end{array}
$$

Then it follows that $v$ does not depend on $x_{1}$ from (1.2), $(\boldsymbol{u} \cdot \nabla) \boldsymbol{u}=\mathbf{0}$ and $p$ depends only on $t$ and $x_{1}$ from (1.1). Therefore we obtain the problem

$$
\begin{gathered}
\frac{\partial v}{\partial t}-v \Delta v=-\frac{\partial p}{\partial x_{1}} \quad \text { in } \quad \boldsymbol{R} \times \Sigma, \\
v=0 \quad \text { on } \quad \boldsymbol{R} \times \partial \Sigma
\end{gathered}
$$

with the time periodic condition

$$
v(t)=v(t+T) \quad \text { in } \quad \Sigma \quad(t \in \boldsymbol{R})
$$

and the flux condition

$$
\int_{\Sigma} v(t) d S=\alpha(t) \quad(t \in \boldsymbol{R})
$$

where $\Delta=\partial^{2} / \partial x_{2}^{2}(n=2), \Delta=\partial^{2} / \partial x_{2}^{2}+\partial^{2} / \partial x_{3}^{2}(n=3)$. We can consider the corresponding problem also in $\boldsymbol{R}^{2}$. We recall that, by appealing to the ideas introduced [4], Galdi and Robertson [8] showed that, for $n=2$, the axial pressure gradient and the flow rate are connected through a simple relation.

It is easy to see that $v$ does not depend on $x_{1}$ and $p$ depends only on $t$ and $x_{1}$. Therefore it follows from the equation (1.6) that $\partial v / \partial t-v \Delta v$ and $\partial p / \partial x_{1}$ depends only on $t$. Moreover, we assume that

$$
p\left(t, x_{1}\right)=\psi(t) x_{1},
$$

where $\psi=\psi(t)$ is the unknown function. Integrating (1.6) on $\Sigma$, we obtain

$$
\psi(t)=-\frac{1}{|\Sigma|}\left(\alpha^{\prime}(t)-v \int_{\Sigma} \Delta v(t) d S\right)
$$

where $|\Sigma|$ is the Lebesgue measure of $\Sigma$. Therefore there exists a time periodic solution $\boldsymbol{u}$ of the Navier-Stokes equations (1.1)-(1.5) in $\omega$, with the form $\boldsymbol{u}=(v, 0)$ or $\boldsymbol{u}=(v, 0,0)$, if and only if $v$ is a solution of the problem

$$
v^{\prime}+v A v-\frac{v}{|\Sigma|}(A v, e) e=\frac{\alpha^{\prime}}{|\Sigma|} e
$$

with the time periodic condition

$$
v(t)=v(t+T) \quad(t \in \boldsymbol{R})
$$


and the flux condition

$$
(v(t), e)=\alpha(t) \quad(t \in \boldsymbol{R}),
$$

where $e(y)=1(y \in \Sigma), A=-\Delta$ with the domain $D(A)=H^{2}(\Sigma) \cap H_{0}^{1}(\Sigma),(v, e)=$ $\int_{\Sigma}$ ved $S$.

Before stating the time periodic result, we introduce the function space. Let $X$ be a Banach space. We set

$$
\begin{aligned}
H_{\pi}^{1}(\boldsymbol{R}) & =\left\{\varphi \in H_{\mathrm{loc}}^{1}(\boldsymbol{R}) ; \varphi(t)=\varphi(t+T) \text { a.e. } t \in \boldsymbol{R}\right\}, \\
L_{\pi}^{2}(\boldsymbol{R} ; X) & =\left\{\varphi \in L_{\mathrm{loc}}^{2}(\boldsymbol{R} ; X) ; \varphi(t)=\varphi(t+T) \text { in } X \text { for a.e. } t \in \boldsymbol{R}\right\}, \\
C_{\pi}(\boldsymbol{R} ; X) & =\{\varphi \in C(\boldsymbol{R} ; X) ; \varphi(t)=\varphi(t+T) \text { in } X \text { for } t \in \boldsymbol{R}\} .
\end{aligned}
$$

Beirão da Veiga [4] proved that for $n \geq 2$ if a flux $\alpha \in H_{\pi}^{1}(\boldsymbol{R})$ is given, then there exists a unique time periodic solution $v^{\alpha}$ of this problem (1.10)-(1.12) satisfying

$$
\begin{aligned}
v^{\alpha} & \in L_{\pi}^{2}\left(\boldsymbol{R} ; H_{0}^{1}(\Sigma) \cap H^{2}(\Sigma)\right) \cap C_{\pi}\left(\boldsymbol{R} ; H_{0}^{1}(\Sigma)\right), \\
\left(v^{\alpha}\right)^{\prime} & \in L_{\pi}^{2}\left(\boldsymbol{R} ; L^{2}(\Sigma)\right) .
\end{aligned}
$$

In this paper, we consider the case $n=2$ and $n=3$.

We apply the regularity result for the heat equations to $v^{\alpha}$. Therefore it is easy to prove that

$$
\begin{aligned}
v^{\alpha} & \in L_{\pi}^{2}\left(\boldsymbol{R} ; H_{0}^{1}(\Sigma) \cap H^{n+1}(\Sigma)\right) \cap C_{\pi}\left(\boldsymbol{R} ; H_{0}^{1}(\Sigma)\right), \\
\left(v^{\alpha}\right)^{\prime} & \in L_{\pi}^{2}\left(\boldsymbol{R} ; H^{n-1}(\Sigma)\right) .
\end{aligned}
$$

Set

$$
\begin{array}{ll}
\boldsymbol{V}^{\alpha}(t, x)=\left(v^{\alpha}(t, x), 0\right) & (n=2), \\
\boldsymbol{V}^{\alpha}(t, x)=\left(v^{\alpha}(t, x), 0,0\right) & (n=3) .
\end{array}
$$

In this paper, let us call $\boldsymbol{V}^{\alpha}$ "the time periodic Poiseuille flow".

2. Time periodic problem in a perturbed channel. In the case $n=2$, for $a_{i}>$ $0(i=1,2)$ we set $\Sigma_{i}:=\left(-a_{i}, a_{i}\right)$. In the case $n=3$, we define $\Sigma_{i}$ as a bounded smooth simply connected domain in $\boldsymbol{R}^{2}$. We write

$$
\omega_{i}=\boldsymbol{R} \times \Sigma_{i} \quad(i=1,2) .
$$

In the channel $\omega_{i}$, if a flux $\alpha \in H_{\pi}^{1}(\boldsymbol{R})$ is given, then there exists a unique solution $v_{i}^{\alpha}$ of the time periodic problem (1.10)-(1.12) on $\Sigma_{i}$. Set

$$
\begin{array}{ll}
\boldsymbol{V}_{i}^{\alpha}(t, x)=\left(v_{i}^{\alpha}(t, x), 0\right) & (n=2), \\
\boldsymbol{V}_{i}^{\alpha}(t, x)=\left(v_{i}^{\alpha}(t, x), 0,0\right) & (n=3) .
\end{array}
$$

For a certain $L>0$ we set

$$
\begin{aligned}
& \omega_{01}=\left\{x \in \omega_{1} ; x_{1} \leq-L\right\}, \\
& \omega_{02}=\left\{x \in \omega_{2} ; x_{1} \geq L\right\} .
\end{aligned}
$$




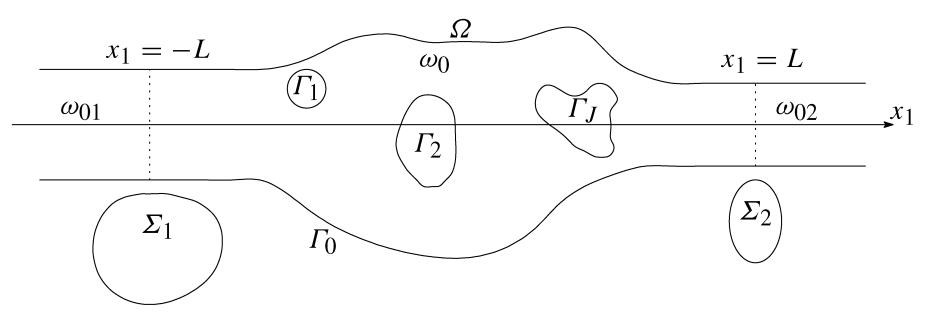

FIGURE 1.

Let $\Omega$ a smooth and unbounded domain in $\boldsymbol{R}^{n}(n=2,3)$ and $\partial \Omega$ a boundary of the domain $\Omega$. We suppose

$$
\begin{aligned}
& \Omega \cap \omega_{01}=\omega_{01}, \\
& \Omega \cap \omega_{02}=\omega_{02} .
\end{aligned}
$$

Let $\omega_{0}$ be $\Omega \backslash\left(\omega_{01} \cup \omega_{02}\right)$. $\omega_{0}$ is a perturbed and bounded part, $\omega_{01}$ and $\omega_{02}$ are channel parts. The boundary $\partial \Omega$ of $\Omega$ has connected components $\Gamma_{0}, \Gamma_{1}, \ldots, \Gamma_{J}$ of $C^{\infty}$-surface such that $\Gamma_{1}, \ldots, \Gamma_{J}$ lie inside of $\Gamma_{0}$ with $\Gamma_{i} \cap \Gamma_{j}=\emptyset$ for $i \neq j$, and such that $\partial \Omega=\bigcup_{j=0}^{J} \Gamma_{j}$. Let us call the domain $\Omega$ "a perturbed channel". See the Figure 1 .

In this paper, for a perturbed channel $\Omega$ we consider the Navier-Stokes equations

$$
\begin{gathered}
\frac{\partial \boldsymbol{u}}{\partial t}-v \Delta \boldsymbol{u}+(\boldsymbol{u} \cdot \nabla) \boldsymbol{u}+\nabla p=\boldsymbol{f} \quad \text { in } \quad(0, T) \times \Omega, \\
\operatorname{div} \boldsymbol{u}=0 \quad \text { in } \quad(0, T) \times \Omega
\end{gathered}
$$

with the boundary condition

$$
\begin{gathered}
\boldsymbol{u}=\mathbf{0} \text { on }(0, T) \times \partial \Omega \\
\boldsymbol{u} \rightarrow \boldsymbol{V}_{i}^{\alpha} \quad \text { as } \quad|x| \rightarrow \infty \text { in } \omega_{0 i} \quad(i=1,2)
\end{gathered}
$$

and the time periodic condition

$$
\boldsymbol{u}(0)=\boldsymbol{u}(T) \text { in } \Omega,
$$

where $\boldsymbol{u}=\boldsymbol{u}(t, x)$ and $p=p(t, x)$ are the unknown velocity and the unknown pressure of an incompressible viscous fluid in $\Omega$, respectively, while $v>0$ is the kinematic viscosity and $f$ is the prescribed external force.

We will seek a solution of (2.1)-(2.5) of the form

$$
\boldsymbol{u}=\boldsymbol{v}+\boldsymbol{V}^{\alpha},
$$

where the velocity field $\boldsymbol{V}^{\alpha}$ is to be such that

$$
\begin{aligned}
\operatorname{div} V^{\alpha} & =0 & & \text { in } \quad \Omega, \\
\boldsymbol{V}^{\alpha} & =\mathbf{0} & & \text { on } \quad \partial \Omega, \\
\boldsymbol{V}^{\alpha} & =\boldsymbol{V}_{i}^{\alpha} & & \text { in } \quad \omega_{0 i} \quad(i=1,2),
\end{aligned}
$$




$$
\begin{aligned}
& \boldsymbol{V}^{\alpha}(t)=\boldsymbol{V}_{i}^{\alpha}(t+T) \quad \text { in } \quad \Omega \quad(t \in \boldsymbol{R}), \\
& \left.\boldsymbol{V}^{\alpha}\right|_{\omega_{0}} \in H_{\pi}^{1}\left((0, T) ; \boldsymbol{H}^{1}\left(\omega_{0}\right)\right),
\end{aligned}
$$

where $\boldsymbol{H}^{1}\left(\omega_{0}\right)=\left\{H^{1}\left(\omega_{0}\right)\right\}^{n}(n=2,3)$. Let us call $\boldsymbol{V}^{\alpha}$ "the extended time periodic Poiseuille flow".

Beirão da Veiga [4] treated the above time periodic problem. He proved that there exists a time periodic solution with the time periodic Poiseuille flow under the restricted flux condition

$$
C(\Omega) \sqrt{v+v^{-2}}\|\alpha\|_{H^{1}(0, T)} \leq \frac{1}{2} v,
$$

where the constant $C(\Omega)$ depends on $\Omega$.

Amick [2] obtaind the following stationary result. $S$ is the two or three dimensional unbounded straight cylinder, where in the case $n=3$ any cross section $\Sigma$ of $S$ is a circle. $V$ is the classical stationary Poiseuille flow in $S$, so it does not depend on the time. We set

$$
\sigma(V)=\sup _{\varphi \in \boldsymbol{H}_{0, \sigma}^{1}(S)} \frac{((\boldsymbol{\varphi} \cdot \nabla) \boldsymbol{\varphi}, V)_{S}}{\|\nabla \boldsymbol{\varphi}\|_{2, S}^{2}} .
$$

The constant $\sigma(V)$ depends only on the cylinder $S$ and the classical Poiseuille flow $V$. Amick [2] proved that in a two and three dimensional perturbed cylinderical domain, there exists a stationary solution with the classical Poiseuille flow $V$ under the restricted flux condition

$$
\sigma(V)<v .
$$

This result is applied to the time periodic problem under the same inequality (2.7) by Kobayashi [13].

In this paper the above upper bound (2.7) is replaced by a more general, and natural, upper bound, where roughly speaking, in definition (2.6) the classical Poiseuille flow $V$ will be replaced by the time periodic Poiseuille flow $\boldsymbol{V}^{\alpha}(t)$. Such a constant denotes $\hat{\gamma}^{\alpha}$. For the details, see Definitions 3.2 and 3.3. If

$$
\hat{\gamma}^{\alpha}<v,
$$

we prove that for the perturbed channel $\Omega$ there exists a time periodic weak solution of the Navier-Stokes equations with the time periodic Poiseuille flow.

Before stating our results we introduce some function spaces. $C_{0, \sigma}^{\infty}(\Omega)$ is the set of all real smooth vector functions with compact support in $\Omega$ such that $\operatorname{div} \varphi=0 . \boldsymbol{L}_{\sigma}^{2}(\Omega)$ is the closure of $\boldsymbol{C}_{0, \sigma}^{\infty}(\Omega)$ in $\boldsymbol{L}^{2}(\Omega)$. The $\boldsymbol{L}^{2}$ inner product and norm on $\Omega$ are denoted as $(\cdot, \cdot)$ and $\|\cdot\|_{2}$, respectively. $\boldsymbol{H}_{0}^{1}(\Omega)$ and $\boldsymbol{H}_{0, \sigma}^{1}(\Omega)$ are the closures of $\boldsymbol{C}_{0}^{\infty}(\Omega)$ and $\boldsymbol{C}_{0, \sigma}^{\infty}(\Omega)$ in $\boldsymbol{H}^{1}(\Omega)$, respectively. $\boldsymbol{H}_{0}^{1}(\Omega)$ and $\boldsymbol{H}_{0, \sigma}^{1}(\Omega)$ are the Hilbert spaces with respect to the inner product $((\boldsymbol{u}, \boldsymbol{v}))=(\nabla \boldsymbol{u}, \nabla \boldsymbol{v})$.

Let $X$ be a Banach space. $C([0, T] ; X), L^{2}((0, T) ; X), L^{\infty}((0, T) ; X)$ and $H^{1}((0, T)$; $X)$ are the usual Banach spaces. $C_{\pi}([0, T] ; X)$ and $H_{\pi}^{1}((0, T) ; X)$ are the sets of all the $C([0, T] ; X)$ and $H_{\pi}^{1}((0, T) ; X)$ functions, respectively, satisfying the time periodic condition $\boldsymbol{u}(0)=\boldsymbol{u}(T)$ in $X$. 
3. Results. Our definition of a time periodic weak solution of the Navier-Stokes equations (2.1)-(2.5) is as follows.

Definition 3.1. A measurable function $\boldsymbol{u}=\boldsymbol{u}(t, x)$ in $(0, T) \times \Omega$ is called a time periodic weak solution of the Navier-Stokes equations if $\boldsymbol{u}$ satisfies the following condition.

(1) $\boldsymbol{v}:=\boldsymbol{u}-\boldsymbol{V}^{\alpha} \in L^{2}\left((0, T) ; \boldsymbol{H}_{0, \sigma}^{1}(\Omega)\right) \cap L^{\infty}\left((0, T) ; \boldsymbol{L}_{\sigma}^{2}(\Omega)\right)$.

(2) $\boldsymbol{u}$ satisfies

$$
\begin{aligned}
\int_{0}^{T}- & (\boldsymbol{u}, \boldsymbol{\varphi}) \psi^{\prime}+\{v(\nabla \boldsymbol{u}, \nabla \boldsymbol{\varphi})+((\boldsymbol{u} \cdot \nabla) \boldsymbol{u}, \boldsymbol{\varphi})\} \psi d t \\
& =\int_{0}^{T}{ }_{\left(\boldsymbol{H}_{0, \sigma}^{1}\right)^{\prime}}\langle\boldsymbol{f}, \boldsymbol{\varphi}\rangle_{\boldsymbol{H}_{0, \sigma}^{1}} \psi d t \quad\left(\boldsymbol{\varphi} \in \boldsymbol{H}_{0, \sigma}^{1}(\Omega), \psi \in C_{0}^{\infty}(0, T)\right) .
\end{aligned}
$$

(3) $\boldsymbol{v}$ is time periodic in $\boldsymbol{L}^{2}(\Omega)$, that is to say, $\boldsymbol{v}$ satisfies

$$
\boldsymbol{v}(0)=\boldsymbol{v}(T) \text { in } \boldsymbol{L}^{2}(\Omega) .
$$

In the straight channel $\omega_{i}$ (not $\Omega$ ), we define a constant concerning the time periodic Poiseuille flow.

DEFINITION 3.2. We set

$$
\gamma_{i}^{\alpha}(t):=\sup _{\varphi \in \boldsymbol{H}_{0, \sigma}^{1}\left(\omega_{i}\right)} \frac{\left((\boldsymbol{\varphi} \cdot \nabla) \boldsymbol{\varphi}, \boldsymbol{V}_{i}^{\alpha}(t)\right)_{\omega_{i}}}{\|\nabla \boldsymbol{\varphi}\|_{2, \omega_{i}}^{2}} \quad(i=1,2, t \in[0, T]) .
$$

For the detailed property of $\gamma_{i}^{\alpha}(t)$, see Proposition 4.1.

DEFINITION 3.3. We set

$$
\begin{aligned}
& \hat{\gamma}_{i}^{\alpha}:=\sup _{t \in[0, T]} \gamma_{i}^{\alpha}(t) \quad(i=1,2), \\
& \hat{\gamma}^{\alpha}:=\max \left\{\hat{\gamma}_{1}^{\alpha}, \hat{\gamma}_{2}^{\alpha}\right\} .
\end{aligned}
$$

Our main theorem on the existence of a time periodic weak solution now reads.

THEOREM 3.1. Suppose that $\boldsymbol{f} \in L^{2}\left((0, T) ;\left(\boldsymbol{H}_{0, \sigma}^{1}(\Omega)\right)^{\prime}\right)$ and $\hat{\gamma}^{\alpha}<v$. Then there exists a time periodic weak solution.

REMARK 3.1. In this paper, we assume that the semi-infinite channels $\omega_{01}$ and $\omega_{02}$ are parallel to the $x_{1}$-axis. But the inequality $\hat{\gamma}^{\alpha}<v$ implies that these semi-infinite channels may not be parallel.

REMARK 3.2. In this paper, the domain $\Omega$ has two outlets $\omega_{01}$ and $\omega_{02}$. We can solve the $J$ outlets problem applying the similar inequality. We consider a straight channel $\omega_{i}=$ $\boldsymbol{R} \times \Sigma_{i}(i=1, \ldots, J, J \geq 3)$, where $\Sigma_{i}$ is a cross section as Section 1. For a given flux function $\alpha_{i} \in H_{\pi}^{1}(\boldsymbol{R})$ and in $\omega_{i}$, we have the time periodic Poiseuille flow $\boldsymbol{V}_{i}^{\alpha_{i}}$. We assume that $\alpha_{i}$ satisfies $\sum_{i=1}^{J} \alpha_{i}(t)=0(t \in \boldsymbol{R})$. We suppose that $\Omega$ has $J$ outlets $\omega_{0 i}(i=1, \ldots, J)$ where $\omega_{0 i}$ is a semi-infinite channel with the cross section $\Sigma_{i}$. In the domain $\Omega$, we consider a time periodic problem with the time periodic Poiseuille flow $\boldsymbol{V}_{i}^{\alpha_{i}}$. We define constant $\hat{\gamma}=$ 
$\max _{1 \leq i \leq J}\left\{\hat{\gamma}_{i}^{\alpha_{i}}\right\}$ as Definitions 3.2 and 3.3. Suppose that $\hat{\gamma}<v$. Then there exists a time periodic weak solution in $\Omega$.

4. The detailed property of $\gamma_{i}^{\alpha}(t)$. In this section we show the property of the $\gamma_{i}^{\alpha}(t)$.

Proposition 4.1. Let $\alpha, \beta \in H_{\pi}^{1}(\boldsymbol{R})$. Then we have

(1) $\gamma_{i}^{\alpha} \in H_{\pi}^{1}(\boldsymbol{R})$,

(2) $\gamma_{i}^{\alpha}(t)>0$,

(3) $\gamma_{i}^{\lambda \alpha}(t)=|\lambda| \gamma_{i}^{\alpha}(t)$,

(4) $\gamma_{i}^{\alpha+\beta}(t)=\gamma_{i}^{\alpha}(t)+\gamma_{i}^{\beta}(t)$.

By this proposition, Definition 3.2 is meaningful.

4.1. Proof of Proposition 4.1. Let us prove (1). It is easy to see that, for any $|h|<1$ and $\boldsymbol{\varphi} \in \boldsymbol{H}_{0, \sigma}^{1}\left(\omega_{i}\right)$, the inequality

$$
\frac{\left((\boldsymbol{\varphi} \cdot \nabla) \boldsymbol{\varphi}, \boldsymbol{V}_{i}^{\alpha}(t+h)\right)_{\omega_{i}}-\left((\boldsymbol{\varphi} \cdot \nabla) \boldsymbol{\varphi}, \boldsymbol{V}_{i}^{\alpha}(t)\right)_{\omega_{i}}}{\|\nabla \boldsymbol{\varphi}\|_{2, \omega_{i}}^{2}} \leq C\left\|v_{i}^{\alpha}(t+h)-v_{i}^{\alpha}(t)\right\|_{H^{n-1}\left(\Sigma_{i}\right)}
$$

holds true, where $C$ depends on Sobolev's Imbedding Theorem $H^{n-1}\left(\Sigma_{i}\right) \hookrightarrow L^{\infty}\left(\Sigma_{i}\right)$ and the Poincaré inequality in $\omega_{i}$. Therefore it follows that

$$
\left|\gamma_{i}^{\alpha}(t+h)-\gamma_{i}^{\alpha}(t)\right| \leq C\left\|v_{i}^{\alpha}(t+h)-v_{i}^{\alpha}(t)\right\|_{H^{n-1}\left(\Sigma_{i}\right)} .
$$

Since $v^{\alpha} \in H^{1}\left((0, T) ; H^{n-1}\left(\Sigma_{i}\right)\right)$, for any interval $(a, b) \subset(0, T)$ we have

$$
\int_{a}^{b} \frac{\left\|v_{i}^{\alpha}(t+h)-v_{i}^{\alpha}(t)\right\|_{H^{n-1}\left(\Sigma_{i}\right)}^{2}}{h} d t \leq C \int_{0}^{T}\left\|\left(v_{i}^{\alpha}\right)^{\prime}(t)\right\|_{H^{n-1}\left(\Sigma_{i}\right)}^{2} d t .
$$

Hence it follows from (4.1) that $\gamma_{i}^{\alpha} \in H^{1}(0, T)$.

Let us prove (2). Let $\boldsymbol{\varphi}$ be $\boldsymbol{H}_{0, \sigma}^{1}\left(\omega_{i}\right)$. Set $\boldsymbol{\xi}(x)=\boldsymbol{\varphi}(-x)$. Then $\boldsymbol{\xi} \in \boldsymbol{H}_{0, \sigma}^{1}\left(\omega_{i}\right)$. We have by a direct calculation

$$
\frac{\left((\boldsymbol{\varphi} \cdot \nabla) \boldsymbol{\varphi}, \boldsymbol{V}_{i}^{\alpha}(t)\right)_{\omega_{i}}}{\|\nabla \boldsymbol{\varphi}\|_{2, \omega_{i}}^{2}}=-\frac{\left((\boldsymbol{\xi} \cdot \nabla) \boldsymbol{\xi}, \boldsymbol{V}_{i}^{\alpha}(t)\right)_{\omega_{i}}}{\|\nabla \boldsymbol{\xi}\|_{2, \omega_{i}}^{2}} .
$$

Therefore we get the inequality

$$
-\gamma_{i}^{\alpha}(t) \leq \frac{\left((\boldsymbol{\varphi} \cdot \nabla) \boldsymbol{\varphi}, \boldsymbol{V}_{i}^{\alpha}(t)\right)_{\omega_{i}}}{\|\nabla \boldsymbol{\varphi}\|_{2, \omega_{i}}^{2}} \leq \gamma_{i}^{\alpha}(t) .
$$

This proves that $\gamma_{i}^{\alpha}(t) \geq 0$. Now we assume that

$$
\gamma_{i}^{\alpha}(t)=0 \text {. }
$$

It follows from (4.2) that

$$
\left((\boldsymbol{\varphi} \cdot \nabla) \boldsymbol{\varphi}, \boldsymbol{V}_{i}^{\alpha}(t)\right)_{\omega_{i}}=0 \quad\left(\boldsymbol{\varphi} \in \boldsymbol{H}_{0, \sigma}^{1}\left(\omega_{i}\right)\right) .
$$

Now for any $\psi \in C_{0}^{\infty}\left(\omega_{i}\right)$, we set

$$
\boldsymbol{\varphi}=\left(\frac{\partial \psi}{\partial x_{2}},-\frac{\partial \psi}{\partial x_{1}}\right) \quad \text { in } \quad \omega_{i} \quad(n=2)
$$




$$
\boldsymbol{\varphi}=\left(\frac{\partial \psi}{\partial x_{2}},-\frac{\partial \psi}{\partial x_{1}}, 0\right) \quad \text { in } \quad \omega_{i} \quad(n=3)
$$

Then $\boldsymbol{\varphi} \in \boldsymbol{C}_{0, \sigma}^{\infty}\left(\omega_{i}\right)$. It follows that

$$
0=\left((\boldsymbol{\varphi} \cdot \nabla) \boldsymbol{\varphi}, \boldsymbol{V}_{i}^{\alpha}(t)\right)_{\omega_{i}}=\int_{\omega_{i}} \psi \frac{\partial^{3} \psi}{\partial x_{1} \partial x_{2}^{2}} v_{i}^{\alpha} d x
$$

It implies that

$$
\frac{\partial^{3} \psi}{\partial x_{1} \partial^{2} x_{2}}=0 \text { in } \omega_{i}
$$

Since $\psi \in C_{0}^{\infty}\left(\omega_{i}\right)$ is an arbitrary function, this is a contradiction. This proves (2).

Let us prove (3). We know that $\lambda v_{i}^{\alpha}$ and $v_{i}^{\lambda \alpha}$ are solutions of (1.10)-(1.11) with the flux condition $\lambda \alpha$. Therefore we have $\lambda \boldsymbol{V}_{i}^{\alpha}=\boldsymbol{V}_{i}^{\lambda \alpha}$. It implies that $\gamma_{i}^{\lambda \alpha}(t)=\lambda \gamma_{i}^{\alpha}(t)$ for any $\lambda>0$. Let $\boldsymbol{\varphi}$ be $\boldsymbol{H}_{0, \sigma}^{1}\left(\omega_{i}\right)$. Set $\boldsymbol{\xi}(x)=\boldsymbol{\varphi}(-x)$. Then $\boldsymbol{\xi} \in \boldsymbol{H}_{0, \sigma}^{1}\left(\omega_{i}\right)$. We obtain

$$
\frac{\left((\boldsymbol{\varphi} \cdot \nabla) \boldsymbol{\varphi}, \boldsymbol{V}_{i}^{\lambda \alpha}(t)\right)_{\omega_{i}}}{\|\nabla \boldsymbol{\varphi}\|_{2, \omega_{i}}^{2}}=-\lambda \frac{\left((\boldsymbol{\xi} \cdot \nabla) \boldsymbol{\xi}, \boldsymbol{V}_{i}^{\alpha}(t)\right)_{\omega_{i}}}{\|\nabla \boldsymbol{\xi}\|_{2, \omega_{i}}^{2}}
$$

by a direct calculation. It implies that $\gamma_{i}^{\lambda \alpha}(t)=-\lambda \gamma_{i}^{\alpha}(t)$ for any $\lambda<0$.

Let us prove (4). We know that $v_{i}^{\alpha+\beta}$ and $v_{i}^{\alpha}+v_{i}^{\beta}$ are solutions of (1.10)-(1.11) with the flux condition $\alpha+\beta$. Therefore we have $V_{i}^{\alpha+\beta}=V_{i}^{\alpha}+V_{i}^{\beta}$. It implies the equality $\gamma_{i}^{\alpha+\beta}(t)=\gamma_{i}^{\alpha}(t)+\gamma_{i}^{\beta}(t)$.

\section{Preliminary.}

5.1. Lemma. In this subsection we show some useful inequlity for the proof of the existence of a time periodic weak solution.

LEMMA 5.1. Let $\boldsymbol{u} \in \boldsymbol{H}_{0}^{1}(\Omega)$. Then

$$
\begin{aligned}
\|\boldsymbol{u}\|_{\boldsymbol{L}^{4}(\Omega)}^{2} & \leq 2^{1 / 2}\|\boldsymbol{u}\|_{2}\|\nabla \boldsymbol{u}\|_{2} \quad(n=2), \\
\|\boldsymbol{u}\|_{\boldsymbol{L}^{4}(\Omega)}^{2} & \leq 2\|\boldsymbol{u}\|_{2}^{1 / 2}\|\nabla \boldsymbol{u}\|_{2}^{3 / 2} \quad(n=3) .
\end{aligned}
$$

LeMmA 5.2. Let $\boldsymbol{u} \in \boldsymbol{H}_{0, \sigma}^{1}(\Omega)$ and $\boldsymbol{v}, \boldsymbol{w} \in \boldsymbol{H}^{1}(\Omega)$. Then

$$
\begin{aligned}
& ((\boldsymbol{u} \cdot \nabla) \boldsymbol{v}, \boldsymbol{w})=-((\boldsymbol{u} \cdot \nabla) \boldsymbol{w}, \boldsymbol{v}), \\
& ((\boldsymbol{u} \cdot \nabla) \boldsymbol{v}, \boldsymbol{v})=0 .
\end{aligned}
$$

Moreover, let $\boldsymbol{v}, \boldsymbol{w} \in \boldsymbol{H}_{0}^{1}(\Omega)$. Then

$$
|((\boldsymbol{u} \cdot \nabla) \boldsymbol{v}, \boldsymbol{w})| \leq C\|\nabla \boldsymbol{u}\|_{2}\|\nabla \boldsymbol{v}\|_{2}\|\nabla \boldsymbol{w}\|_{2} .
$$

LEMMA 5.3. For any $\varepsilon>0$ and $\boldsymbol{w} \in C\left([0, T] ; \boldsymbol{L}^{n}(\Omega)\right)(n=2,3)$, there exist an integer $N$ and functions $\psi_{j} \in \boldsymbol{L}^{2}(\Omega)(j=1, \ldots, N)$ such that

$$
\int_{0}^{T}|((\boldsymbol{u} \cdot \nabla) \boldsymbol{v}, \boldsymbol{w})| d t \leq \varepsilon \int_{0}^{T}\left(\|\nabla \boldsymbol{u}\|_{2}^{2}+\|\nabla \boldsymbol{v}\|_{2}^{2}+\|\boldsymbol{u}\|_{2}\|\nabla \boldsymbol{v}\|_{2}\right) d t+\sum_{j=1}^{N} \int_{0}^{T}\left|\left(\boldsymbol{u}, \boldsymbol{\psi}_{j}\right)\right|^{2} d t
$$


for any $\boldsymbol{u}, \boldsymbol{v} \in L^{2}\left((0, T) ; \boldsymbol{H}_{0, \sigma}^{1}(\Omega)\right)$.

For the proof of this lemma, see Masuda [17, Lemma 2.5].

5.2. Auxiliary proposition for the extended time periodic flow. The following propositions are improved for the time periodic Poiseuille flow. For the original propositions, see Amick [2].

For a fixed $t \in[0, T]$ we define a functional $\boldsymbol{r}(t)$ as

$$
\boldsymbol{\varphi} \in \boldsymbol{H}_{0, \sigma}^{1}(\Omega) \mapsto\left(\left(\boldsymbol{V}^{\alpha}\right)^{\prime}(t), \boldsymbol{\varphi}\right)+v\left(\nabla \boldsymbol{V}^{\alpha}(t), \nabla \boldsymbol{\varphi}\right)+\left(\left(\boldsymbol{V}^{\alpha}(t) \cdot \nabla\right) \boldsymbol{V}^{\alpha}(t), \boldsymbol{\varphi}\right) .
$$

Then we have the following proposition.

Proposition 5.1. The map $\boldsymbol{r}(t)$ is a linear and continuous functional on $\boldsymbol{H}_{0, \sigma}^{1}(\Omega)$. Furthermore we have ${ }_{\left(\boldsymbol{H}_{0, \sigma}^{1}\right)^{\prime}}\langle\boldsymbol{r}, \boldsymbol{\varphi}\rangle_{\boldsymbol{H}_{0, \sigma}^{1}} \in L^{2}(0, T)$ for any $\boldsymbol{\varphi} \in \boldsymbol{H}_{0, \sigma}^{1}(\Omega)$.

For the proof of this proposition, see Amick [2, Lemma 3.4].

Suppose that $\theta \in C^{\infty}(\boldsymbol{R})$ satisfies

$$
\begin{aligned}
0 \leq \theta(s) \leq 1 & (s \in \boldsymbol{R}), \\
\theta(s)=1 & (s \leq-1), \\
\theta(s)=0 & (s \geq 0) .
\end{aligned}
$$

For any $\delta>0$, we set

$$
\theta_{\delta}(x)= \begin{cases}\theta\left(\delta x_{1}\right) & \left(x \in \omega_{01}\right), \\ \theta\left(-\delta x_{1}\right) & \left(x \in \omega_{02}\right), \\ 0 & \text { otherwise }\end{cases}
$$

Then we have the following proposition.

Proposition 5.2. For any $\varepsilon>0$, there exists an $\boldsymbol{s} \in H_{\pi}^{1}\left((0, T) ; \boldsymbol{H}_{0, \sigma}^{1}(\Omega)\right)$ with compact support such that the inequality

$$
\left((\boldsymbol{v} \cdot \nabla) \boldsymbol{v}, \boldsymbol{V}^{\alpha}\right) \leq((\boldsymbol{v} \cdot \nabla) \boldsymbol{v}, \boldsymbol{s})+\left((\boldsymbol{v} \cdot \nabla) \boldsymbol{v}, \boldsymbol{V}^{\alpha} \theta_{\delta}^{2}\right)+\left(\varepsilon+c_{0} \delta\right)\|\nabla \boldsymbol{v}\|_{2}^{2} \quad\left(\boldsymbol{v} \in \boldsymbol{H}_{0, \sigma}^{1}(\Omega)\right)
$$

holds true, where the constant $c_{0}$ does not depend on $\varepsilon$ and $\delta$.

For the proof of this proposition, see Amick [2, p.495-p.496].

We set

$$
\Gamma_{\delta}(t):=\sup _{v \in \boldsymbol{H}_{0, \sigma}^{1}(\Omega)} \frac{\left((\boldsymbol{v} \cdot \nabla) \boldsymbol{v}, \boldsymbol{V}^{\alpha}(t) \theta_{\delta}^{2}\right)}{\|\nabla \boldsymbol{v}\|_{2, \Omega}^{2}} .
$$

Then we have the following proposition.

Proposition 5.3. We have

$$
\lim _{\delta \rightarrow+0} \Gamma_{\delta}(t)=\max \left\{\gamma_{1}(t), \gamma_{2}(t)\right\} .
$$

For the proof of this proposition, see Amick [2, Theorem 4.3]. 


\section{Proof of Theorem 3.1.}

6.1. Time periodic weak solution in a bounded domain. In this section, we prove Theorem 3.1 for a three dimensional domain. The proof for a two dimensional domain is similar.

We consider that a sequence of bounded domains $\Omega^{n}(n \in N)$ such that $\omega_{0} \subset \Omega^{1}$, $\Omega^{n} \subset \Omega^{n+1}, \bigcup_{n \in N} \Omega^{n}=\Omega$ and $\partial \Omega^{n}$ is of class $C^{\infty}$. Let $\Gamma_{0}^{n}$ be the outer boundary of $\Omega^{n}$.

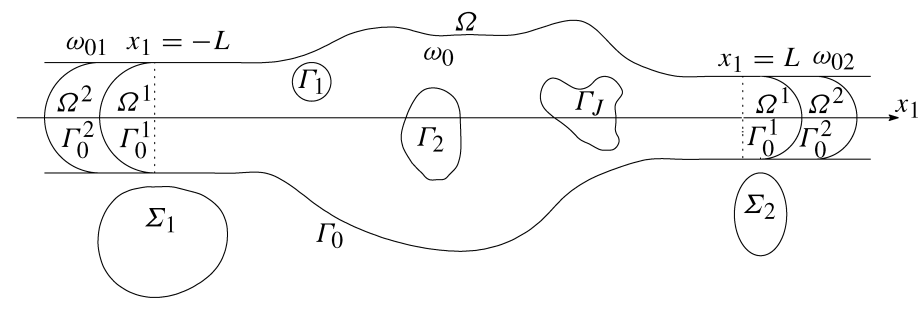

FIGURE 2.

In the bounded domain $\Omega^{n}$, we consider the time periodic problems of the Navier-Stokes equations with the Dirichlet boundary condition

$$
\begin{aligned}
& \frac{\partial \boldsymbol{u}}{\partial t}-v \Delta \boldsymbol{u}+(\boldsymbol{u} \cdot \nabla) \boldsymbol{u}+\nabla p=\boldsymbol{f} \quad \text { in } \quad(0, T) \times \Omega^{n}, \\
& \operatorname{div} \boldsymbol{u}=0 \quad \text { in } \quad(0, T) \times \Omega^{n}, \\
& \boldsymbol{u}=\boldsymbol{V}^{\alpha} \quad \text { on } \quad(0, T) \times \partial \Omega^{n}, \\
& \boldsymbol{u}(0)=\boldsymbol{u}(T) \text { in } \Omega^{n} \text {. }
\end{aligned}
$$

Precisely, the boundary value $\left.\boldsymbol{V}^{\alpha}\right|_{\partial \Omega^{n}}$ satisfies

$$
\begin{aligned}
\left.\boldsymbol{V}^{\alpha}\right|_{\partial \Omega^{n}} & \in H_{\pi}^{1}\left((0, T) ; \boldsymbol{H}^{\frac{1}{2}}\left(\partial \Omega^{n}\right)\right), \\
\int_{\Gamma_{0}^{n}} \boldsymbol{V}^{\alpha} \cdot \boldsymbol{n} d S & =0, \\
\boldsymbol{V}^{\alpha} & =\mathbf{0} \quad \text { on } \quad \Gamma_{j} \quad(j=1, \ldots, J) .
\end{aligned}
$$

Therefore there exists a solenoidal $\boldsymbol{b}_{\varepsilon}^{n} \in H_{\pi}^{1}\left((0, T) ; \boldsymbol{H}^{1}\left(\Omega^{n}\right)\right)$ such that

$$
\boldsymbol{b}_{\varepsilon}^{n}=\boldsymbol{V}^{\alpha} \quad \text { on } \quad(0, T) \times \partial \Omega^{n},
$$

Here, we obtained the inequality (6.1) for three dimensional bounded domains. Corresponding results in dimension two are given in Kobayashi [14], Lemma 2.6. From (6.1), there exists a solenoidal $\boldsymbol{u}_{n} \in L^{2}\left((0, T) ; \boldsymbol{H}^{1}\left(\Omega^{n}\right)\right) \cap L^{\infty}\left((0, T) ; \boldsymbol{L}_{\sigma}^{2}\left(\Omega^{n}\right)\right)$ such that

$$
\frac{d}{d t}\left(\boldsymbol{u}_{n}, \boldsymbol{\varphi}\right)_{n}+v\left(\nabla \boldsymbol{u}_{n}, \nabla \boldsymbol{\varphi}\right)_{n}+\left(\left(\boldsymbol{u}_{n} \cdot \nabla\right) \boldsymbol{u}_{n}, \boldsymbol{\varphi}\right)_{n}=\langle\boldsymbol{f}, \boldsymbol{\varphi}\rangle \quad\left(\boldsymbol{\varphi} \in \boldsymbol{V}\left(\Omega^{n}\right)\right),
$$

$$
\boldsymbol{u}_{n}=\boldsymbol{V}^{\alpha} \quad \text { on } \quad(0, T) \times \partial \Omega^{n} \quad \text { (in the trace sense) },
$$




$$
\boldsymbol{u}_{n}(0)=\boldsymbol{u}_{n}(T) \text { in } \boldsymbol{L}^{2}\left(\Omega^{n}\right),
$$

where $(\cdot, \cdot)_{n}$ denotes the $\boldsymbol{L}^{2}$ inner product on $\Omega^{n}$. For the proof, see Yudović [26].

6.2. The estimate of an initial value. In the pervious subsection, for the bounded domain $\Omega^{n}$ we obtain the time periodic solution $\boldsymbol{u}_{n}$. Set

$$
\boldsymbol{v}_{n}=\left\{\begin{array}{lll}
\boldsymbol{u}_{n}-\boldsymbol{V}^{\alpha} & \text { in } & \Omega^{n}, \\
\mathbf{0} & \text { in } & \Omega \backslash \Omega^{n} .
\end{array}\right.
$$

In this subsection, we will prove that $\left\|\boldsymbol{v}_{n}(0)\right\|_{2, \Omega}$ is bounded with respect to $n$.

It follows that

$$
\begin{aligned}
& \boldsymbol{v}_{n} \in L^{2}\left((0, T) ; \boldsymbol{H}_{0, \sigma}^{1}(\Omega)\right) \cap L^{\infty}\left((0, T) ; \boldsymbol{L}_{\sigma}^{2}(\Omega)\right), \\
& \left(\boldsymbol{v}_{n}\right)^{\prime} \in L^{1}\left((0, T) ;\left(\boldsymbol{H}_{0, \sigma}^{1}(\Omega)\right)^{\prime}\right), \\
& \frac{d}{d t}\left(\boldsymbol{v}_{n}, \boldsymbol{\varphi}\right)+v\left(\left(\boldsymbol{v}_{n}, \boldsymbol{\varphi}\right)\right)+\left(\left(\boldsymbol{v}_{n} \cdot \nabla\right) \boldsymbol{v}_{n}, \boldsymbol{\varphi}\right)+\left(\left(\boldsymbol{v}_{n} \cdot \nabla\right) \boldsymbol{V}^{\alpha}, \boldsymbol{\varphi}\right)+\left(\left(\boldsymbol{V}^{\alpha} \cdot \nabla\right) \boldsymbol{v}, \boldsymbol{\varphi}\right) \\
& =\langle\boldsymbol{G}, \boldsymbol{\varphi}\rangle,
\end{aligned}
$$

$$
\boldsymbol{v}_{n}(0)=\boldsymbol{v}_{n}(T) \text { in } \boldsymbol{L}^{2}(\Omega),
$$

where $\boldsymbol{\varphi}$ is extended as a $\mathbf{0}$ function on the outside $\Omega^{n}$,

$$
\langle\boldsymbol{G}, \boldsymbol{\varphi}\rangle=\langle\boldsymbol{f}, \boldsymbol{\varphi}\rangle-\left(\left(\boldsymbol{V}^{\alpha}\right)^{\prime}, \boldsymbol{\varphi}\right)-v\left(\nabla \boldsymbol{V}^{\alpha}, \nabla \boldsymbol{\varphi}\right)-\left(\left(\boldsymbol{V}^{\alpha} \cdot \nabla\right) \boldsymbol{V}^{\alpha}, \boldsymbol{\varphi}\right) \quad\left(\boldsymbol{\varphi} \in \boldsymbol{H}_{0, \sigma}^{1}(\Omega)\right) .
$$

Setting $\varphi=\boldsymbol{v}_{n}$ in (6.5), we obtain

$$
\frac{1}{2} \frac{d}{d t}\left\|\boldsymbol{v}_{n}\right\|_{2}^{2}+v\left\|\nabla \boldsymbol{v}_{n}\right\|_{2}^{2}=\left(\left(\boldsymbol{v}_{n} \cdot \nabla\right) \boldsymbol{v}_{n}, \boldsymbol{V}^{\alpha}\right)+\left\langle\boldsymbol{G}, \boldsymbol{v}_{n}\right\rangle .
$$

We apply Proposition 5.2 to $\left(\left(\boldsymbol{v}_{n} \cdot \nabla\right) \boldsymbol{v}_{n}, \boldsymbol{V}^{\alpha}\right)$. Then for any $\varepsilon>0$ and $\delta>0$, there exists an $s \in H_{\pi}^{1}\left((0, T) ; \boldsymbol{H}_{0, \sigma}^{1}(\Omega)\right)$ with compact support such that

$$
\left(\left(\boldsymbol{v}_{n} \cdot \nabla\right) \boldsymbol{v}_{n}, \boldsymbol{V}^{\alpha}\right) \leq\left(\left(\boldsymbol{v}_{n} \cdot \nabla\right) \boldsymbol{v}_{n}, \boldsymbol{s}\right)+\left(\left(\boldsymbol{v}_{n} \cdot \nabla\right) \boldsymbol{v}_{n}, \boldsymbol{V}^{\alpha} \theta_{\delta}^{2}\right)+\left(\varepsilon+c_{0} \delta\right)\left\|\nabla \boldsymbol{v}_{n}\right\|_{2}^{2} .
$$

Furthermore, it follows that

$$
\left(\left(\boldsymbol{v}_{n} \cdot \nabla\right) \boldsymbol{v}_{n}, \boldsymbol{V}^{\alpha} \theta_{\delta}^{2}\right)=\frac{\left(\left(\boldsymbol{v}_{n} \cdot \nabla\right) \boldsymbol{v}_{n}, \boldsymbol{V}^{\alpha} \theta_{\delta}^{2}\right)}{\left\|\nabla \boldsymbol{v}_{n}\right\|_{2}^{2}}\left\|\nabla \boldsymbol{v}_{n}\right\|_{2}^{2} \leq \Gamma_{\delta}(t)\left\|\nabla \boldsymbol{v}_{n}\right\|_{2}^{2} .
$$

We choose $\varepsilon>0$ such that

$$
v-\hat{\gamma}^{\alpha}-4 \varepsilon>0 .
$$

Applying Proposition 5.3 to $\Gamma_{\delta}(t)$, we can choose $\delta>0$ such that

$$
\Gamma_{\delta}(t) \leq \hat{\gamma}^{\alpha}+\varepsilon .
$$

Moreover, we choose $\delta>0$ such that

$$
v-\hat{\gamma}^{\alpha}-4 \varepsilon-c_{0} \delta>0 .
$$

It holds that

$$
\frac{1}{2} \frac{d}{d t}\left\|\boldsymbol{v}_{n}\right\|_{2}^{2}+\left(v-\hat{\gamma}^{\alpha}-3 \varepsilon-c_{0} \delta\right)\left\|\nabla \boldsymbol{v}_{n}\right\|_{2}^{2} \leq\left(\left(\boldsymbol{v}_{n} \cdot \nabla\right) \boldsymbol{v}_{n}, \boldsymbol{s}\right)+C\|\boldsymbol{G}\|_{\left(\boldsymbol{H}_{0, \sigma}^{1}\right)^{\prime}}^{2},
$$


where the constant $C$ depends only on $\varepsilon$. There exists an $N \in N$ such that $\operatorname{supp}(\varphi) \subset \Omega^{n}$ for all $n \geq N$. We may set $\varphi=s$ in (6.5). Since

$$
\frac{d}{d t}\left(\boldsymbol{v}_{n}, \boldsymbol{s}\right)=\left\langle\boldsymbol{v}_{n}^{\prime}, \boldsymbol{s}\right\rangle+\left(\boldsymbol{v}_{n}, \boldsymbol{s}^{\prime}\right),
$$

we have

$$
\begin{aligned}
\left(\left(\boldsymbol{v}_{n} \cdot \nabla\right) \boldsymbol{v}_{n}, \boldsymbol{s}\right)= & -\frac{d}{d t}\left(\boldsymbol{v}_{n}, \boldsymbol{s}\right)-\left(\boldsymbol{v}_{n}, \boldsymbol{s}^{\prime}\right)-v\left(\left(\boldsymbol{v}_{n}, \boldsymbol{s}\right)\right) \\
& -\left(\left(\boldsymbol{v}_{n} \cdot \nabla\right) \boldsymbol{V}^{\alpha}, \boldsymbol{s}\right)-\left(\left(\boldsymbol{V}^{\alpha} \cdot \nabla\right) \boldsymbol{v}_{n}, \boldsymbol{s}\right)+\langle\boldsymbol{G}, \boldsymbol{s}\rangle \\
\leq & -\frac{d}{d t}\left(\boldsymbol{v}_{n}, \boldsymbol{s}\right)+\varepsilon\left\|\nabla \boldsymbol{v}_{n}\right\|_{2}^{2}+C\left(\|\nabla \boldsymbol{s}\|_{2}^{2}+\left\|\boldsymbol{s}^{\prime}\right\|_{2}^{2}+\|\boldsymbol{G}\|_{\left(\boldsymbol{H}_{0, \sigma}^{1}\right)^{\prime}}^{2}\right),
\end{aligned}
$$

where the constant $C$ depnds on $\boldsymbol{V}^{\alpha}, \varepsilon$ and the Poincaré inequality. We define $K_{1}(t)$ by

$$
K_{1}(t)=C\left(\|\nabla \boldsymbol{s}(t)\|_{2}^{2}+\left\|\boldsymbol{s}^{\prime}(t)\right\|_{2}^{2}+\|\boldsymbol{G}(t)\|_{\left(\boldsymbol{H}_{0, \sigma}^{1}\right)^{\prime}}^{2}\right) .
$$

Then it follows that

$$
\frac{1}{2} \frac{d}{d t}\left\|\boldsymbol{v}_{n}\right\|_{2}^{2}+\left(v-\hat{\gamma}^{\alpha}-4 \varepsilon-c_{0} \delta\right)\left\|\nabla \boldsymbol{v}_{n}\right\|_{2}^{2} \leq-\frac{d}{d t}\left(\boldsymbol{v}_{n}, \boldsymbol{s}\right)+K_{1} .
$$

Applying the Poincaré inequality to the second term of the left-hand side of (6.9), we have

$$
\frac{d}{d t}\left\|\boldsymbol{v}_{n}\right\|_{2}^{2}+\mu\left\|\boldsymbol{v}_{n}\right\|_{2}^{2} \leq-2 \frac{d}{d t}\left(\boldsymbol{v}_{n}, \boldsymbol{s}\right)+2 K_{1}
$$

where

$$
\mu=\frac{2\left(v-\hat{\gamma}^{\alpha}-4 \varepsilon-c_{0} \delta\right)}{C(\Omega)^{2}} .
$$

For a certain $\xi>0$ (smaller than $\mu$ ), multiplying (6.10) by $e^{(\mu-\xi) t}$, we obtain

$$
\begin{aligned}
e^{(\mu-\xi) t} & \frac{d}{d t}\left\|\boldsymbol{v}_{n}(t)\right\|_{2}^{2}+\mu e^{(\mu-\xi) t}\left\|\boldsymbol{v}_{n}(t)\right\|_{2}^{2} \\
& \leq-2 e^{(\mu-\xi) t} \frac{d}{d t}\left(\boldsymbol{v}_{n}(t), \boldsymbol{s}(t)\right)+2 K_{1}(t) e^{(\mu-\xi) t} \\
& =-2 \frac{d}{d t}\left\{\left(\boldsymbol{v}_{n}(t), \boldsymbol{s}(t)\right) e^{(\mu-\xi) t}\right\}+2(\mu-\xi) e^{(\mu-\xi) t}\left(\boldsymbol{v}_{n}(t), \boldsymbol{s}(t)\right)+2 K_{1}(t) e^{(\mu-\xi) t} \\
& \leq-2 \frac{d}{d t}\left\{\left(\boldsymbol{v}_{n}(t), \boldsymbol{s}(t)\right) e^{(\mu-\xi) t}\right\}+\xi e^{(\mu-\xi) t}\left\|\boldsymbol{v}_{n}(t)\right\|_{2}^{2}+\left(C\|\boldsymbol{s}(t)\|_{2}^{2}+2 K_{1}(t)\right) e^{(\mu-\xi) t},
\end{aligned}
$$

where the constant $C$ depends only on $\mu$ and $\xi$. Indeed, it follows from (6.11) that

$$
\frac{d}{d t}\left(e^{(\mu-\xi) t}\left\|\boldsymbol{v}_{n}(t)\right\|_{2}^{2}\right) \leq-2 \frac{d}{d t}\left\{\left(\boldsymbol{v}_{n}(t), \boldsymbol{s}(t)\right) e^{(\mu-\xi) t}\right\}+K_{2}(t),
$$

where

$$
K_{2}(t)=\left(C\|s(t)\|_{2}^{2}+2 K_{1}(t)\right) e^{(\mu-\xi) t} .
$$

Integrating (6.12) on $[0, T]$, we have

$$
\left\|\boldsymbol{v}_{n}(T)\right\|_{2}^{2} e^{(\mu-\xi) T} \leq\left\|\boldsymbol{v}_{n}(0)\right\|_{2}^{2}-2\left(\boldsymbol{v}_{n}(T), s(T)\right) e^{(\mu-\xi) T}+2\left(\boldsymbol{v}_{n}(0), s(0)\right)+K,
$$


where

$$
K=\int_{0}^{T} K_{2}(t) d t
$$

Since $\boldsymbol{v}_{n}$ and $s$ are time periodic in $\boldsymbol{L}^{2}(\Omega)$, for any $\lambda>0$ we have

$$
\begin{aligned}
\left\|\boldsymbol{v}_{n}(0)\right\|_{2}^{2} e^{(\mu-\xi) T} \leq & \left\|\boldsymbol{v}_{n}(0)\right\|_{2}^{2}+\left(\lambda\left\|\boldsymbol{v}_{n}(0)\right\|_{2}^{2}+C\|\boldsymbol{s}(0)\|_{2}^{2}\right) e^{(\mu-\xi) T} \\
& +\lambda\left\|\boldsymbol{v}_{n}(0)\right\|_{2}^{2}+C\|\boldsymbol{s}(0)\|_{2}^{2}+K,
\end{aligned}
$$

where the constant $C$ depends only on $\lambda$. We set

$$
\begin{aligned}
H & =K e^{-(\mu-\xi) T}+C\|s(0)\|_{2}^{2}\left(e^{-(\mu-\xi) T}+1\right), \\
\theta & =1-\lambda-(1+\lambda) e^{-(\mu-\xi) T} .
\end{aligned}
$$

We choose $\lambda>0$ such that $\theta$ is greater than 0 . Then we have

$$
\left\|\boldsymbol{v}_{n}(0)\right\|_{2}^{2} \leq \frac{H}{\theta}=: M_{0}
$$

Consequetly, the sequence $\left\|\boldsymbol{v}_{n}(0)\right\|_{2}$ is bounded with respect to $n$.

6.3. A priori estimate and weak limit. In this subsection, we prove that $\boldsymbol{v}_{n}$ is a bounded sequence in $L^{2}\left((0, T) ; \boldsymbol{H}_{0, \sigma}^{1}(\Omega)\right) \cap L^{\infty}\left((0, T) ; \boldsymbol{L}_{\sigma}^{2}(\Omega)\right)$.

It follows from the equation (6.7) that

$$
\begin{aligned}
\frac{1}{2} \frac{d}{d t}\left\|\boldsymbol{v}_{n}\right\|_{2}^{2}+v\left\|\nabla \boldsymbol{v}_{n}\right\|_{2}^{2} & =\left(\left(\boldsymbol{v}_{n} \cdot \nabla\right) \boldsymbol{v}_{n}, \boldsymbol{V}^{\alpha}\right)+\left\langle\boldsymbol{G}, \boldsymbol{v}_{n}\right\rangle \\
& \leq C\left(\boldsymbol{V}^{\alpha}\right)\left\|\boldsymbol{v}_{n}\right\|_{2}\left\|\nabla \boldsymbol{v}_{n}\right\|_{2}+\|\boldsymbol{G}\|_{\left(\boldsymbol{H}_{0, \sigma}^{1}\right)^{\prime}}\left\|\nabla \boldsymbol{v}_{n}\right\|_{2} \\
& \leq \frac{C_{1}}{2}\left\|\boldsymbol{v}_{n}\right\|_{2}^{2}+\frac{v}{2}\left\|\nabla \boldsymbol{v}_{n}\right\|_{2}^{2}+\frac{C_{2}}{2}\|\boldsymbol{G}\|_{\left(\boldsymbol{H}_{0, \sigma}^{1}\right)^{\prime}}^{2},
\end{aligned}
$$

where the constant $C_{1}$ depends only on $\boldsymbol{V}^{\alpha}$ and $\nu$, the constant $C_{2}$ depends only on $v$. So it follows

$$
\frac{d}{d t}\left\|\boldsymbol{v}_{n}\right\|_{2}^{2}+v\left\|\nabla \boldsymbol{v}_{n}\right\|_{2}^{2} \leq C_{1}\left\|\boldsymbol{v}_{n}\right\|_{2}+C_{2}\|\boldsymbol{G}\|_{\left(\boldsymbol{H}_{0, \sigma}^{1}\right)^{\prime}} .
$$

Applying the Gronwall inequality to (6.13) and integrating from 0 to $t(\leq T)$, we obtain

$$
\left\|\boldsymbol{v}_{n}(t)\right\|_{2}^{2} \leq M_{0} e^{C_{1} T}+C_{2} \int_{0}^{T} e^{C_{1} t}\|\boldsymbol{G}\|_{\left(\boldsymbol{H}_{0, \sigma}^{1}\right)^{\prime}}^{2} d t=: M_{1} .
$$

Integrating (6.13) on $[0, T]$, we deduce the inequality

$$
\int_{0}^{T}\left\|\nabla \boldsymbol{v}_{n}\right\|_{2}^{2} d t \leq \frac{1}{v}\left(C_{1} T M_{1}+C_{2} \int_{0}^{T}\|\boldsymbol{G}\|_{\left(H_{0, \sigma}^{1}\right)^{\prime}}^{2} d t\right)=: M_{2} .
$$

For each $\varphi \in C_{0, \sigma}^{\infty}(\Omega)$, we choose $J \in N$ such that $\operatorname{supp}(\varphi) \subset \Omega_{n}$ for any $n \geq J$. Then we have

$$
\left|\left(\boldsymbol{v}_{n}(t), \varphi\right)\right| \leq\left\|\boldsymbol{v}_{n}(t)\right\|_{2}\|\varphi\|_{2} \leq M_{1}\|\varphi\|_{2}
$$

and

$$
\left|\left(\boldsymbol{v}_{n}(t), \boldsymbol{\varphi}\right)-\left(\boldsymbol{v}_{n}(s), \boldsymbol{\varphi}\right)\right|
$$




$$
\begin{aligned}
& =\left|\int_{s}^{t} \frac{d}{d \tau}\left(\boldsymbol{v}_{n}(\tau), \boldsymbol{\varphi}\right) d \tau\right| \\
& \leq \int_{s}^{t} v\left|\left(\left(\boldsymbol{v}_{n}, \boldsymbol{\varphi}\right)\right)\right|+\left|\left(\left(\boldsymbol{v}_{n} \cdot \nabla\right) \boldsymbol{v}_{n}, \boldsymbol{\varphi}\right)\right|+\left|\left(\left(\boldsymbol{v}_{n} \cdot \nabla\right) \boldsymbol{V}^{\alpha}, \boldsymbol{\varphi}\right)\right|+\left|\left(\left(\boldsymbol{V}^{\alpha} \cdot \nabla\right) \boldsymbol{v}_{n}, \boldsymbol{\varphi}\right)\right|+|\langle\boldsymbol{G}, \boldsymbol{\varphi}\rangle| d \tau \\
& \leq \int_{s}^{t}\left(v\left\|\nabla \boldsymbol{v}_{n}\right\|_{2}+2\left\|\boldsymbol{v}_{n}\right\|_{2}^{1 / 2}\left\|\nabla \boldsymbol{v}_{n}\right\|_{2}^{3 / 2}+2 C\left(\boldsymbol{V}^{\alpha}\right)\left\|\nabla \boldsymbol{v}_{n}\right\|_{2}+\|\boldsymbol{G}\|_{\left(\boldsymbol{H}_{0, \sigma}^{1}\right)^{\prime}}\right)\|\nabla \boldsymbol{\varphi}\|_{2} d \tau \\
& \leq\left(M_{3}|t-s|^{1 / 2}+M_{4}|t-s|^{1 / 4}\right)\|\nabla \boldsymbol{\varphi}\|_{2},
\end{aligned}
$$

where the constant $M_{3}$ and $M_{4}$ do not depend on $n$. Therefore $\left\{\left(\boldsymbol{v}_{n}(t), \varphi\right)\right\}_{n \geq J}$ is uniformly bounded and equicontinuous on $[0, T]$.

Since $\left\{\boldsymbol{v}_{n}\right\}$ is a bounded sequence in $L^{2}\left((0, T) ; \boldsymbol{H}_{0, \sigma}^{1}(\Omega)\right) \cap L^{\infty}\left((0, T) ; \boldsymbol{L}_{\sigma}^{2}(\Omega)\right)$, there exists a subsequence $\left\{\boldsymbol{v}_{n, k}\right\}_{k}$ of $\left\{\boldsymbol{v}_{n}\right\}$ and $\boldsymbol{v} \in L^{2}\left((0, T) ; \boldsymbol{H}_{0, \sigma}^{1}(\Omega)\right) \cap L^{\infty}\left((0, T) ; \boldsymbol{L}_{\sigma}^{2}(\Omega)\right)$ such that

$$
\boldsymbol{v}_{n, k} \rightarrow \boldsymbol{v} \quad(k \rightarrow \infty) \quad \text { in } \quad \begin{cases}L^{\infty}\left((0, T) ; \boldsymbol{L}_{\sigma}^{2}(\Omega)\right) & \text { weak star } \\ L^{2}\left((0, T) ; \boldsymbol{H}_{0, \sigma}^{1}(\Omega)\right) & \text { weakly }\end{cases}
$$

For any $\boldsymbol{\varphi} \in \boldsymbol{C}_{0, \sigma}^{\infty}(\Omega)$, there exists a subsequence $\left\{\boldsymbol{v}_{n, k, i}\right\}$ of $\left\{\boldsymbol{v}_{n, k}\right\}$ such that

$$
\left(\boldsymbol{v}_{n, k, i}, \boldsymbol{\varphi}\right)=(\boldsymbol{v}, \boldsymbol{\varphi}) \quad \text { uniformly } \quad \text { on }[0, T] \quad(i \rightarrow \infty)
$$

holds by the Ascoli-Arzelá Theorem.

We will prove that the convergence (6.15) holds for any $\boldsymbol{\varphi} \in \boldsymbol{L}^{2}(\Omega)$. We have the orthogonal decomposition

$$
\boldsymbol{\varphi}=\boldsymbol{\varphi}_{\sigma}+\boldsymbol{\varphi}_{p} \quad\left(\boldsymbol{\varphi}_{\sigma} \in \boldsymbol{L}_{\sigma}^{2}(\Omega), \boldsymbol{\varphi}_{p} \in\left(\boldsymbol{L}_{\sigma}^{2}(\Omega)\right)^{\perp}\right) .
$$

Since $\boldsymbol{C}_{0, \sigma}^{\infty}(\Omega)$ is dence in $\boldsymbol{L}_{\sigma}^{2}(\Omega)$, for any $\eta>0$ there exists a $\boldsymbol{\varphi}_{\sigma}^{\eta} \in \boldsymbol{C}_{0, \sigma}^{\infty}(\Omega)$ such that

$$
\left\|\varphi_{\sigma}^{\eta}-\boldsymbol{\varphi}_{\sigma}\right\|_{2}<\eta \text {. }
$$

We have

$$
\begin{aligned}
\left|\left(\boldsymbol{v}-\boldsymbol{v}_{n}, \boldsymbol{\varphi}\right)\right| & \leq\left|\left(\boldsymbol{v}-\boldsymbol{v}_{n}, \boldsymbol{\varphi}_{\sigma}-\boldsymbol{\varphi}_{\sigma}^{\eta}\right)\right|+\left|\left(\boldsymbol{v}-\boldsymbol{w}_{n}, \boldsymbol{\varphi}_{\sigma}^{\eta}\right)\right| \\
& \leq 2 M_{1} \eta+\left|\left(\boldsymbol{v}-\boldsymbol{w}_{n}, \boldsymbol{\varphi}_{\sigma}^{\eta}\right)\right|
\end{aligned}
$$

because $\boldsymbol{v}_{n}$ is bounded in $L^{\infty}\left((0, T) ; \boldsymbol{L}_{\sigma}^{2}(\Omega)\right)$. We can choose a subsequence $\left\{\boldsymbol{v}_{n, k}\right\}_{k}$ of $\left\{\boldsymbol{v}_{n}\right\}_{n}$ such that the second term of the right-hand side of (6.16) goes to 0 . Therefore, for any $\varphi \in$ $\boldsymbol{L}^{2}(\Omega)$ there exists a subsequence $\left\{\boldsymbol{v}_{n, k, i}\right\}$ such that $\left(\boldsymbol{v}_{n, k, i}, \boldsymbol{\varphi}\right)$ converges to $(\boldsymbol{v}, \boldsymbol{\varphi})$ uniformly on $[0, T]$.

6.4. Time periodic weak solution. In this subsection we prove that $\boldsymbol{u}=\boldsymbol{v}+\boldsymbol{V}^{\alpha}$ is a time periodic weak solution.

For any $\varphi \in C_{0, \sigma}^{\infty}(\Omega)$, we choose $J \in N$ such that $\operatorname{supp}(\varphi) \subset \Omega^{n}$ for any $n \geq J$. We mulitiply (6.5) by $\psi \in C_{0}^{\infty}(0, T)$ and integrate on $[0, T]$. Then we have

$$
\begin{aligned}
& \int_{0}^{T}-\left(\boldsymbol{v}_{n}, \boldsymbol{\varphi}\right) \psi^{\prime}+\left\{v\left(\left(\boldsymbol{v}_{n}, \boldsymbol{\varphi}\right)\right)+\left(\left(\boldsymbol{v}_{n} \cdot \nabla\right) \boldsymbol{v}_{n}, \boldsymbol{\varphi}\right)\right. \\
&\left.\left.+\left(\left(\boldsymbol{v}_{n} \cdot \nabla\right) \boldsymbol{V}^{\alpha}\right), \boldsymbol{\varphi}\right)+\left(\left(\boldsymbol{V}^{\alpha} \cdot \nabla\right) \boldsymbol{v}_{n}, \boldsymbol{\varphi}\right)\right\} \psi d t
\end{aligned}
$$




$$
=\int_{0}^{T}\langle\boldsymbol{G}, \boldsymbol{\varphi}\rangle \psi d t
$$

It follows from (6.14) that there exists a subsequence $\left\{\boldsymbol{v}_{n, k}\right\}_{k \in N}$ such that the left-hand side of (6.17) except the nonliner term converges to

$$
\left.\int_{0}^{T}-(\boldsymbol{v}, \boldsymbol{\varphi}) \psi^{\prime}+\left\{v((\boldsymbol{v}, \boldsymbol{\varphi}))+\left((\boldsymbol{v} \cdot \nabla) \boldsymbol{V}^{\alpha}\right), \boldsymbol{\varphi}\right)+\left(\left(\boldsymbol{V}^{\alpha} \cdot \nabla\right) \boldsymbol{v}, \boldsymbol{\varphi}\right)\right\} \psi d t .
$$

We prove that there exists a subsequence $\left\{\boldsymbol{v}_{n, k, i}\right\}$ such that

$$
\int_{0}^{T}\left(\left(\boldsymbol{v}_{n, k, i} \cdot \nabla\right) \boldsymbol{v}_{n, k, i}, \boldsymbol{\varphi}\right) \psi d t \rightarrow \int_{0}^{T}((\boldsymbol{v} \cdot \nabla) \boldsymbol{v}, \boldsymbol{\varphi}) \psi d t \quad(i \rightarrow \infty) .
$$

We have

$$
\begin{aligned}
& \int_{0}^{T}\left(\left(\boldsymbol{v}_{n, k} \cdot \nabla\right) \boldsymbol{v}_{n, k}, \boldsymbol{\varphi}\right) \psi d t-\int_{0}^{T}((\boldsymbol{v} \cdot \nabla) \boldsymbol{v}, \boldsymbol{\varphi}) \psi d t \\
& \quad=\int_{0}^{T}\left(\left(\boldsymbol{v}_{n, k}-\boldsymbol{v}\right) \cdot \nabla \boldsymbol{v}_{n, k}, \boldsymbol{\varphi}\right) \psi d t-\int_{0}^{T}\left(\psi \boldsymbol{v} \cdot \nabla \boldsymbol{\varphi}, \boldsymbol{v}_{n, k}-\boldsymbol{v}\right) d t \quad\left(=: I_{1}+I_{2}\right) .
\end{aligned}
$$

Now let us consider $I_{1}$. Applying Lemma 5.3 to $I_{1}$, we see that for any $\eta>0$ there exists an integer $N_{1}$ and $\psi_{l} \in \boldsymbol{L}^{2}(\Omega)\left(l=1, \ldots, N_{1}\right)$ such that

$$
\left|I_{1}\right| \leq M_{5} \eta+\sum_{l=1}^{N_{1}} \int_{0}^{T}\left|\left(\boldsymbol{v}_{n, k}-\boldsymbol{v}, \boldsymbol{\psi}_{l}\right)\right|^{2} d t
$$

where the constant $M_{5}$ does not depend on $n$.

Let us consider $I_{2}$. Since we know that $\psi(t) \boldsymbol{v}(t) \cdot \nabla \boldsymbol{\varphi} \in \boldsymbol{L}^{2}(\Omega)$ for a.e. $t \in(0, T)$, it follows that

$$
\psi(t) \boldsymbol{v}(t) \cdot \nabla \boldsymbol{\varphi}=\boldsymbol{\Phi}_{\sigma}(t)+\boldsymbol{\Phi}_{p}(t) \quad\left(\boldsymbol{\Phi}_{\sigma}(t) \in \boldsymbol{L}_{\sigma}^{2}(\Omega), \boldsymbol{\Phi}_{p}(t) \in\left(\boldsymbol{L}_{\sigma}^{2}(\Omega)\right)^{\perp}\right) .
$$

Since $\boldsymbol{v}_{n, k}(t)-\boldsymbol{v}(t) \in \boldsymbol{L}_{\sigma}^{2}(\Omega)$ for a.e. $t \in(0, T)$, we have

$$
\int_{0}^{T}\left(\psi \boldsymbol{v} \cdot \nabla \boldsymbol{\varphi}, \boldsymbol{v}_{n, k}-\boldsymbol{v}\right) d t=\int_{0}^{T}\left(\boldsymbol{\Phi}_{\sigma}, \boldsymbol{v}_{n, k}-\boldsymbol{v}\right) d t
$$

Consequently, it follows from (6.20) and (6.21) that

$$
\left|I_{1}\right|+\left|I_{2}\right| \leq M_{5} \eta+\sum_{l=1}^{N_{1}} \int_{0}^{T}\left|\left(\boldsymbol{v}_{n, k}-\boldsymbol{v}, \boldsymbol{\psi}_{l}\right)\right|^{2} d t+\left|\int_{0}^{T}\left(\boldsymbol{\Phi}_{\sigma}, \boldsymbol{v}_{n, k}-\boldsymbol{v}\right) d t\right| .
$$

We can choose a subsequence $\left\{\boldsymbol{v}_{n, k, i}\right\}_{i \in N}$ of $\left\{\boldsymbol{v}_{n, k}\right\}_{k \in N}$ such that the second and third terms of the right-hand side of (6.22) converge to 0 from (6.15). This proves the convergence of (6.18).

The above convergence implies that $\boldsymbol{v}$ satisfies

$$
\begin{aligned}
\int_{0}^{T} & -(\boldsymbol{v}, \boldsymbol{\varphi}) \psi^{\prime}+\left\{v((\boldsymbol{v}, \boldsymbol{\varphi}))+((\boldsymbol{v} \cdot \nabla) \boldsymbol{v}, \boldsymbol{\varphi})+\left((\boldsymbol{v} \cdot \nabla) \boldsymbol{V}^{\alpha}, \boldsymbol{\varphi}\right)+\left(\left(\boldsymbol{V}^{\alpha} \cdot \nabla\right) \boldsymbol{v}, \boldsymbol{\varphi}\right)\right\} \psi d t \\
& =\int_{0}^{T}\langle\boldsymbol{G}, \boldsymbol{\varphi}\rangle \psi d t .
\end{aligned}
$$


Since $\boldsymbol{C}_{0, \sigma}^{\infty}(\Omega) \subset \boldsymbol{H}_{0, \sigma}^{1}(\Omega)$ is dense, we have

$$
\begin{aligned}
\int_{0}^{T} & -(\boldsymbol{v}, \boldsymbol{\varphi}) \psi^{\prime}+\left\{v((\boldsymbol{v}, \boldsymbol{\varphi}))+((\boldsymbol{v} \cdot \nabla) \boldsymbol{v}, \boldsymbol{\varphi})+\left((\boldsymbol{v} \cdot \nabla) \boldsymbol{V}^{\alpha}, \boldsymbol{\varphi}\right)+\left(\left(\boldsymbol{V}^{\alpha} \cdot \nabla\right) \boldsymbol{v}, \boldsymbol{\varphi}\right)\right\} \psi d t \\
& =\int_{0}^{T}\langle\boldsymbol{G}, \boldsymbol{\varphi}\rangle \psi d t \quad\left(\boldsymbol{\varphi} \in \boldsymbol{H}_{0, \sigma}^{1}(\Omega), \psi \in C_{0}^{\infty}(0, T)\right) .
\end{aligned}
$$

Lastly, we prove that $\boldsymbol{v}$ is time periodic in $\boldsymbol{L}^{2}(\Omega)$. For any $\boldsymbol{\varphi} \in \boldsymbol{L}^{2}(\Omega)$, there exists a subsequence $\left\{\boldsymbol{v}_{n, k}\right\}$ such that the limit (6.15) holds true. Therefore, it follows that

$$
(\boldsymbol{v}(0)-\boldsymbol{v}(T), \boldsymbol{\varphi})=\left(\boldsymbol{v}(0)-\boldsymbol{v}_{n, k}(0), \varphi\right)+\left(\boldsymbol{v}_{n, k}(T)-\boldsymbol{v}(T), \varphi\right) \rightarrow 0 \quad(k \rightarrow \infty),
$$

that is to say, $\boldsymbol{v}$ is time periodic in $\boldsymbol{L}^{2}(\Omega)$. We set

$$
\boldsymbol{u}=\boldsymbol{v}+\boldsymbol{V}^{\alpha} .
$$

Then $\boldsymbol{u}$ is a time periodic weak solution.

Acknowledgments. The author would like to express to Professor Hiroko Morimoto his deepest gratitude for her unceasing encouragement and valuable advice.

\section{REFERENCES}

[1] R. A. Adamas and J. J. F. Fournier, Sobolev spaces. Second edition, Pure Appl. Math. (Amst.) 140, Elsevier/Academic Press, Amsterdam, 2003.

[2] C. J. AмIск, Steady solutions of the Navier-Stokes equations in unbounded channels and pipes, Ann. Scuola Norm. Sup. Pisa Cl. Sci. (4) 4 (1977), 473-513.

[3] C. J. Аміск, Properties of steady Navier-Stokes solutions for certain unbounded channels and pipes, Nonlinear Anal. 2 (1978), 689-720.

[4] H. BEIRÃO DA VeIgA, Time-periodic solutions of the Navier-Stokes equations in unbounded cylindrical domains-Leray's problem for periodics flows, Arch. Ration. Mech. Anal. 178 (2005), 301-325.

[5] R. FinN, On the steady-state solutions of the Navier-Stokes equations, III, Acta. Math. 105 (1961), 197-244.

[6] H. FujitA, On the existence and regularity of the steady-state solutions of the Navier-Stokes equation, J. Fac. Sci. Univ. Tokyo Sect. I 9 (1961), 59-102.

[7] G. P. GALDI, An Introduction to the mathematical theory of the Navier-Stokes equations, Vol. I, Linearized steady problems, Springer Tracts Nat. Philos. 38, Springer-Verlag, New York, 1994.

[8] G. P. Galdi And A. M. Robertson, The relation between flow rate and axial pressure gradient for timeperiodic Poiseuille Flow in a pipe, J. Math. Fluid. Mech. 7 (2005), suppl. 2, S215-S223.

[9] D. Gilbarg And N. S. Trudinger, Elliptic partial differential equations of second order, Second edition, Grundlehren Math. Wiss. 224, Springer-Verlag, Berlin, 1983.

[10] D. D. Joseph And S. CARmi, Stability of Poiseuille flow in pipes, annuli, and channels, Quart. Appl. Math. 26 (1969), 575-599.

[11] S. Kaniel And M. Shinbrot, A reproductive property of the Navier-Stokes equations, Arch. Rational Mech. Anal. 24 (1967), 363-369.

[12] T. Kоваyashi, Time periodic solutions of the Navier-Stokes equations under general outflow condition, Tokyo J. Math. 32 (2009), no. 2, 409-424.

[13] T. KobAYASHI, The relation between stationary and periodic solutions of the Navier-Stokes equations in two or three dimensional channels, J. Math. Kyoto Univ. 49 (2009), no. 2, 307-323.

[14] T. KовAYASHI, Time periodic solutions of the Navier-Stokes equations under general outflow condition in a two dimensional symmetric channel, Hokkaido Math. J. 39 (2010), no. 3, 291-316. 
[15] O. A. LAdyzhenskaya, The mathematical theory of viscous incompressible flow, Second English edition, revised and enlarged, Gordon and Breach, Science Publishers, New York-London-Paris, 1969.

[16] J. L. LiONS, Quelques méthodes de résolution des problèmes aux limites non linéaries, Dunod, GauthierVillars, Paris, 1969.

[17] K. MAsudA, Weak solutions of Navier-Stokes equations, Tôhoku Math. J. 36 (1984), 623-646.

[18] H. Morimoto AND H. FujitA, A remark on the existence of steady Navier-Stokes flow in 2D semi-infinite channel involving the general outflow condition, Math. Bohem. 126 (2001), no. 2, 457-468.

[19] H. MoRimoto, Stationary Navier-Stokes flow in 2-D channels involving the general outflow condition, Handbook of differential equations, stationary partial differential equations. Vol. IV, 299-353, Handb. Differ. Equ., Elsevier/North-Holland, Amsterdam, 2007.

[20] H. Morimoto, Time periodic Navier-Stokes flow with nonhomogeneous boundary condition, J. Math. Sci. Univ. Tokyo 16 (2009), no. 1, 113-123.

[21] A. TAKeshita, On the reproductive property of the 2-dimensional Navier-Stokes equations, J. Fac. Sci. Univ. Tokyo Sec. IA 16 (1970), 297-311.

[22] K. Pileckas, On nonstationary two-dimensional Leray's problem for Poiseuille flow, Adv. Math. Sci. Appl. 16 (2006), no. 1, 141-174.

[23] V. A. Solonnikov, Solvability of a problem of the flow of a viscous incompressible fluid into an infinite open basin, Proc. Steklov Inst. Math. (1989), no. 2, 193-225.

[24] R. Temam, Navier-Stokes equations, Theory and numerical analysis, With an appendix by F. Thomasset, Third edition, Stud. Math. Appl. 2, North-Holland Publishing Co., Amsterdam, 1984.

[25] K. YosidA, Functional analysis, Third edition, Springer-Verlag, 1980.

[26] V. I. YUdovič, Periodic motions of a viscous incompressible fluid, Soviet Math. Dokl. 1 (1960), 168-172.

Department of Mathematics

MEIJI UNIVERSITY

1-1-1 TAMA-KU, KAWASAKI, 214-0038

JAPAN

E-mail address: teppeik@isc.meiji.ac.jp 